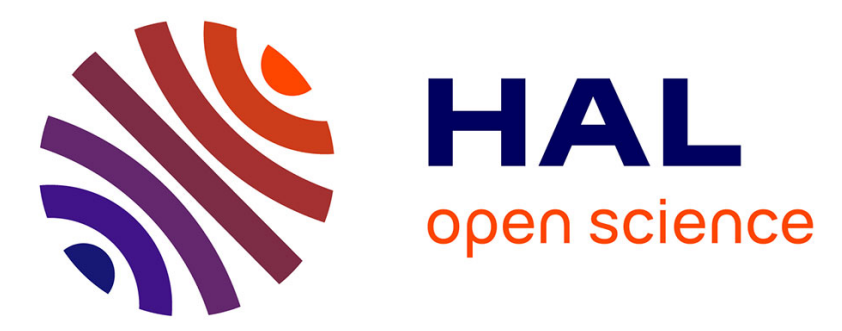

\title{
Marquage vital in utero de tissus calcifiés chez Myocastor coypus
}

\author{
E. Fichet, M. Pascal
}

\section{To cite this version:}

E. Fichet, M. Pascal. Marquage vital in utero de tissus calcifiés chez Myocastor coypus. Mammalia, 1989, 53 (3), pp.451-455. hal-02724810

\section{HAL Id: hal-02724810 \\ https://hal.inrae.fr/hal-02724810}

Submitted on 2 Jun 2020

HAL is a multi-disciplinary open access archive for the deposit and dissemination of scientific research documents, whether they are published or not. The documents may come from teaching and research institutions in France or abroad, or from public or private research centers.
L'archive ouverte pluridisciplinaire HAL, est destinée au dépôt et à la diffusion de documents scientifiques de niveau recherche, publiés ou non, émanant des établissements d'enseignement et de recherche français ou étrangers, des laboratoires publics ou privés. 


\title{
Marquage vital in utero de tissus calcifiés chez Myocastor coypus
}

\author{
par E. FICHET et M. PASCAL
}

\author{
Laboratoire de la Faune Sauvage. I.N.R.A.-C.R.J.J. - F-78350 Jouy-en-Josas
}

Summary. - A community of aquatic wild rodents localizated on the bank of a Loire river tributari was collectively marked by using baits (carots) coated with calcified tissues vital dyes : fluorescein and xylenol orange. An exhaustive trapping operation was undertaken at the end of the baiting time. Among the trapped rodents, we found five pregnant females of nutria (Myocastor coypus) the calcified tissues of which was labelled. The labelling of the fœtal lower incisor dentin of three fœtuses belonging to one female shaws the ability of the xylenol orange to cross by the placental barrier and to mark the fœtal dentine. We suggest the use of this result in the view to resolve some question in relation to population biology.

Résumé. - Un marquage collectif au moyen d'appâts additionnés de fluorochromes, la fluorescéine et le xylénol orange, a été réalisé en Anjou sur un peuplement de rongeurs rivulaires. Au nombre de ces rongeurs figure le ragondin, Myocastor coypus, dont les fœtus de cinq femelles gestantes et marquées sont analysés. Le passage du xylénol orange à travers la barrière placentaire et sa fixation au niveau de la dentine de l'incisive inférieure fœtale est mis en évidence, ouvrant une perspective d'utilisation de cette technique de marquage en biologie des populations.

\section{INTRODUCTION}

L'usage de divers fluoromarqueurs vitaux pour étudier l'ontogenèse des tissus calcifiés est de découverte ancienne (Duhamel 1742) et largement documenté (Bang et Enlow 1967 ; Olerud et Lorenzi 1970 ; Meunier et Boivin 1978 ; Francillon 1980 ; Meunier et Pascal 1981 ; Castanet 1982 ; De Buffrénil et Pascal 1984, inter alia). L'injection constitue le mode d'administration le plus utilisé de ces marqueurs. Cependant, dans la perspective de réaliser des marquages collectifs à l'échelle de la population et sur de vastes surfaces, des écologues ont entrepris d'utiliser et, au préalable, de tester les performances de fixation de ces produits administrés par voie orale (Wandeler et al. 1975 ; Klevezal et Mina 1980 ; Pascal

Mammalia, t. 53, $n^{\circ} 3,1989$. 
et al. 1988 ; Fichet et Pascal 1988). Chez l'homme, des travaux réalisés durant les années 1955-1965, période pendant laquelle l'antibiothérapie prescrite à de nombreuses femmes enceintes comportait des tétracyclines, ont montré que ces molécules pouvaient passer la barrière placentaire (Maynard et al. 1954 ; Posner et al. 1955 ; Febles-Alfonzo et Batthyany 1959) et présenter un danger pour l'embryon (Ibsen et Urist 1964). En outre, elles se fixent dans les tissus dentaire (émail et dentine) et osseux (Dorval et Demers 1968) pour peu que l'antibiotique soit administré à forte dose $(1 \mathrm{~g} / \mathrm{j})$ pendant une période particulière de l'embryogenèse ( 25 à 28 semaines).

Chez le rat, Cahen et Fave (1970) ont montré que l'injection de tétracycline induit un marquage des tissus osseux fœtaux. Des résultats similaires ont été observés chez la ratte en gestation lorsque cet antibiotique est incorporé à la nourriture (Hurley et Tuchman-Duplessis 1963).

A l'occasion d'une expérience de marquage collectif réalisée au moyen d'appâts contenant du xylénol orange ou de la fluorescéine et menée en nature sur un peuplement de rongeurs rivulaires (Fichet et Pascal 1988), nous avons pu mettre en évidence le marquage de la dentine in utero chez le ragondin (Myocastor coypus).

\section{MATÉRIEL ET MÉTHODE}

Les descriptions du site de travail et des protocoles expérimentaux sont consignées dans une précédente publication (Fichet et Pascal 1988). Au nombre des 98 ragondins capturés à l'occasion de cette expérience figuraient 28 femelles en activité sexuelle (gestante et/ou allaitante). Parmi celles-ci, 5 seulẹment présentaient à la fois la caractéristique d'avoir leurs tissus squelettiques marqués par l'un et/ou l'autre des fluoromarqueurs et de porter des embryons suffisamment développés (poids $>70 \mathrm{~g}$ ) pour permettre des prélèvements sur le terrain (Tab. 1).

Une demie mandibule de chaque embryon a été incluse dans une résine polyester (Francillon et Meunier 1985), débitée en lames de 0,2 à 0,5 mm d'épaisseur au moyen d'une scie diamantée, collée sur une lame de verre au moyen d'une colle époxy, amincie jusqu'à 0,06-0,1 mm et observée à trois reprises (Pascal et Castanet 1978) au microscope optique en épifluorescence (Olympus BH2, lampe $100 \mathrm{w}$ mercure) aux grossissements 40 et 100.

\section{RÉSULTATS}

L'ensemble des résultats figure sur le tableau 1. Seule la femelle à la fois doublement marquée et la plus tardivement capturée (au $4^{\mathrm{e}}$ contrôle, contrairement aux autres qui l'ont été au $1^{\text {er }}$ ) portait trois embryons dont la dentine de l'incisive inférieure présentait une coloration orange, témoin du marquage par le xylénol orange (Fig. 1). La spongiosa de la mandibule de ces fotus n'a pas fixé le fluorochrome de façon perceptible. 




Fig. 1. - Localisation du marquage par l'orangé de xylénol (XO) dans la dentine (d) de l'incisive fœtale. li : loge de l'incisive ; e : émail ; cp : cavité pulpaire.

Xylenol orange (XO) labeling localization in foetal incisor dentin (d). li : incisor cavity ; e : enamel ; cp : pulp cavity.

TABLEAU I. - Nature du marqueur (F : fluorescéine, X.O. : orangé de xylénol) identifié chez la mère (M.M.) et chez les fœtus (M.F.). N Fœtus : nombre de fœetus, Pds : poids moyen des fœtus (g), J : durée écoulée en jours entre le début du piégeage et la capture.

\begin{tabular}{llcccc} 
N & J & M.M. & N. Foetus & Pds. & M.F. \\
\hline 410 & 1 & $F$ & 7 & 126 & $-:-$ \\
426 & 1 & F & 6 & 73 & $-:-$ \\
436 & 1 & $F$ & 10 & 130 & $-:-$ \\
438 & 1 & XO & 6 & 206 & $-:-$ \\
485 & 4 & $F+X . O$ & 3 & 132 & $X 0$
\end{tabular}

\section{DISCUSSION - CONCLUSION}

Si les résultats de l'expérience évoquée ci-dessus démontrent clairement la possibilité de réaliser en nature un marquage vital des tissus calcifiés in utero chez le ragondin, ils ne permettent cependant pas de cerner clairement la, ou les causes, à l'origine des quatre échecs enregistrés.

En effet, alors que les tissus calcifiés de la femelle 485 sont marqués par les deux fluorochromes, ceux de ses trois fœtus ne le sont que par le seul xylénol orange. Faut-il y voir une capacité différentielle des deux molécules à passer la barrière placentaire, en rapport avec une différence de poids moléculaire (622 pour la fluorescéine versus, 604 pour l'orangé de xylenol) ou de nature stéréochimi- 
que ? Si tel est strictement le cas, contrairement à ce qui est observé, les fœtus de la femelle 438 marquée au xylénol orange devraient eux aussi être marqués. A une notion qualitative doit donc se superposer une notion quantitative : peut-on identifier des situations qui permettraient aux tissus fœtaux de recevoir plus ou moins de produit marqueur ? Il est vrai, par exemple, que l'effectif de la seule portée marquée est le plus petit du lot expérimental. Il est possible d'imaginer que la quantité de produit amené à chaque embryon sera d'autant plus importante que cet effectif sera réduit. Il est vrai aussi que la femelle porteuse de ces embryons marqués a été capturée la dernière et a donc eu la possibilité d'absorber des appâts plus longtemps et en quantité plus importante que les autres. Enfin, chez l'homme, Toaff en 1966 (in Dorval et Demers 1968) a mis en évidence l'influence du stade embryonnaire sur la fixation des tétracyclines dans le tissu dentaire. Leophonte (1988) affirme, quant à lui, que la diffusion transplacentaire des antibiotiques est maximale au troisième trimestre de gestation chez la femme. Si l'on admet un bon niveau de corrélation entre l'âge des fœetus et leur poids, cet argument n'est pas recevable pour expliquer à lui seul l'absence de marquage chez les embryons de la femelle 438 .

Le bien fondé de ces diverses hypothèses ne pourra être rigoureusement établi qu'à l'issue d'expériences menées en captivité. Il reste cependant possible de tester, à l'occasion d'expériences menées dans la nature, l'effet de l'augmentation du temps d'appâtage sur le taux de marquage embryonnaire. Si ce dernier se révèle satisfaisant et à la condition de vérifier une bonne persistance du marquage post-natal, l'intérêt de l'usage du marquage vital des tissus calcifiés dans une perspective d'étude de la biologie des populations se trouverait considérablement renforcé.

Remerciements. - Ce travail a reçu le soutien financier de la F.D.G.P.C. Maine-etLoire, du Fonds des Calamités agricoles ainsi que de l'A.T.P. C.N.R.S. Biologie des Populations.

\section{BIBLIOGRAPHIE}

BANG, S. and D.H. ENLOw, 1967. - The postnatal growth of the rabbit mandible. Arch. Oral Biol., 12 : 993-998.

CASTANET, J., 1982. - Recherche sur la croissance du tissu osseux des Reptiles. Application : la méthode squelettochronologique. Thèse de Doctorat ès Sciences, Université Paris II. 156 pp.

CAHEN, R. et A. FAVE, 1970. - Y a-t-il une relation entre l'effet tératogène et le passage transplacentaire d'un médicament ? C.R. Séances Soc. Biol., 164 : 610-616.

De Bufrrenil, V. et M. Pascal, 1984. - Morphogenèse post-natale de la mandibule de Vison (Mustela vison Schreiber) : données sur la dynamique et l'interprétation fonctionnelle des dépôts osseux mandibulaires. Can. J. Zool., 62 : 2026-2037.

Dorval, J. et P.P. Demers, 1968. - Les effets secondaires des tétracyclines en pédiatrie. Laval Médical, 39 : 939-954.

Duhamel, M., 1742. - Sur le développement et la crue des os des animaux. Mem. Acad. Roy. Sc. Paris : 364-367. 
Febles-Alfonzo, D. and C. Batthyany, 1959. - An experimental study of oxytetracycline : local genital absorption and diffusion and placental transmission to the blood of the ombilical cord and to the amniotic fluid. Antibiot. Ann. : 846-849.

Fichet, E. et M. PASCAL, 1988. - Marquage collectif de rongeurs sauvages au moyen de fluoromarqueurs vitaux des tissus calcifiés. J. Can. Zool., sous presse.

FranCILlON, H., 1980. - Mise en évidence expérimentale du caractère annuel des lignes d'arrêt de croissance (LAC) chez le Triton crêté, Triturus cristatus (LAUR). Bull. Soc. Zool. Fr., 105 : 243-275.

Francillon, H. et F. Meunier, 1985. - Conservation et présentation des préparations colorées au Bleu Alcian et à l'Alizarine. Cibium, 9 : 121-126.

HuRLeY, L.S. et H. TuChMAN-DuPlesSis, 1963. - Influence de la tétracycline sur le développement pré- et post-natal du rat. C.R. Acad. Sci., 257 (1) : 302-304.

IBSEN, K. and R. URIST, 1964. - The biochemistry and the physiology of the tetracyclines : with special reference to mineralized tissues. Clin. Orthopedics, 32 : 143-169.

KLEVEZAL, G.A. and M.V. MINA, 1980. - A tetracycline method of group marking for rodents and prospect of its utilization in ecological studies (en russe). Zool. Zh., 59 : 936-941.

LEOPHONTE, P., 1988. - Antibiotiques au cours de la grossesse et de l'allaitement : conséquences sur le traitement des infections respiratoires. Rev. Mal. Resp., 5 : 293-298.

Maynard, A. de L., A. Prigot and J.C. Andriola, 1954. - Observation of the absorption, diffusion and excretion of tetracycline hydrochloride. Antibiot. Ann. : 655-658.

MeUnier, F. et G. BoIvin, 1978. - Action de la fluorescéine, de l'alizarine, du bleu de calcéïne et de diverses doses de tétracycline sur la croissance de la Truite et de la Carpe. Ann. Biol. Anim. Bioch. Biophys., $8:$ 1293-1308.

Meunier, F. et M. PASCAL, 1981. - Etude expérimentale de la croissance cyclique des rayons de la nageoire de la Carpe (Cyprinus carpio L.). Résultats préliminaires. Aquaculture, $26: 23-40$.

OleRUD, S. and G.L. LORENZI, 1970. - Triple fluorochrome labelling in bone formation and bone resorption. J. Bone Joint Surg., 52 A (2) : 274-278.

Pascal, M. et L. Castanet, 1978. - Méthode de détermination de l'âge chez le chat haret des îles Kerguelen. Rev. Ecol. Terre et Vie, 32 : 529-555.

Pascal, M., E. Fichet, H. Burin Des Roziers et P. Douville, 1989. - Méthode de marquage des tissus calcifiés du ragondin au moyen d'appâts additionnés de fluoromarqueurs vitaux. Rev. Ecol. Terre et Vie, janvier 1989, sous presse.

Posner, A.C., N.G. Konicoff and A. Prigot, 1955. - Tetracycline in obstetric infections. Antibiot. Ann. : 345-348.

Wandeler, A., P. Pfotenhauer and C. Stocker, 1975. - Über die Verwendung von Ködern zu biologischen Untersuchungen an Füchsen. Rev. Suisse Zool., 82 : 335-348. 